\title{
INTERCULTURALIDAD Y PRÁCTICAS DOCENTES EN CLASES DE HISTORIA, GEOGRAFÍA Y CIENCIAS SOCIALES, EN ESCUELAS CON ALTO PORCENTAJE DE ALUMNOS MIGRANTES DE LA COMUNA DE SANTIAGO ${ }^{1}$
}

\section{Laura Valledor ${ }^{2}$, Luis Garcés ${ }^{3}$, Pablo Whipple ${ }^{4}$}

\begin{abstract}
RESUMEN
Este artículo presenta un estudio exploratorio de las prácticas docentes en la asignatura Historia, Geografía y Ciencias Sociales, de cuatro profesores en escuelas con alta matrícula inmigrante de la comuna de Santiago. El propósito de la investigación fue conocer las respuestas docentes al desafío de enseñar a estudiantes cuyos referentes culturales e historias nacionales son diversos. Se aplicó una metodología cualitativa con enfoque Grounded Theory para generar conocimiento desde las prácticas mismas. Los resultados indican que los docentes aplican fundamentalmente dos estrategias: (1) la comparación entre las características del mismo hecho, proceso histórico o tema en Chile y en los países de origen de los alumnos inmigrantes, y (2) el uso de analogías entre las experiencias de los alumnos inmigrantes y los contenidos obligatorios de la asignatura. Dichas estrategias concuerdan con algunos principios de un enfoque intercultural de la enseñanza, pero coexisten con explicaciones relacionadas con un concepto esencialista de la identidad nacional y narrativas de superioridad cultural. Los hallazgos orientan acerca de los énfasis que debieran tener futuros programas de acompañamiento y formación continua, que ayuden a mejorar las prácticas que los profesores ya están impulsando, y a profundizar el concepto de interculturalidad en la enseñanza de la asignatura.
\end{abstract}

Conceptos clave: prácticas docentes, inmigrantes, interculturalidad, historia y ciencias sociales.

1 Proyecto apoyado financieramente por CNED/ Convocatoria 2018.

2 Pontificia Universidad Católica de Chile, Santiago, Chile. Contacto: 1svalled @uc.cl

3 Pontificia Universidad Católica de Chile, Santiago, Chile. Contacto: legarces@uc.cl

4 Pontificia Universidad Católica de Chile, Santiago, Chile. Contacto: pwhipple@uc.cl 


\title{
INTERCULTURALITY AND TEACHING PRACTICES IN HISTORY, GEOGRAPHY AND SOCIAL SCIENCES IN SCHOOLS WITH A HIGH PERCENTAGE OF MIGRANT STUDENTS IN THE DISTRICT OF SANTIAGO
}

\begin{abstract}
This article presents an exploratory study of four teachers' teaching practices in the subject of History, Geography and Social Sciences, in schools with a high immigrant enrollment, in the district of Santiago. The purpose of this research was to explore how teachers respond to the challenge of teaching students with diverse cultural and historical backgrounds. The study uses a qualitative approach with a Grounded Theory focus, in order to generate knowledge from the practices themselves. The results indicate that teachers essentially apply two strategies in the classroom: (1) comparisons between the characteristics of Chilean events, historical processes or topics, with those of the immigrant students' countries of origin, and (2) the use of analogies between the experiences of immigrant students and the curriculum's mandatory content. Although these strategies coincide with some principles of an intercultural teaching approach, they coexist with explanations related to an essentialist idea of national identity and narratives of cultural superiority. These findings can guide future continuing education and support programs and help teachers to improve the practices they are currently using and to deepen the understanding of interculturality in the teaching of History and Social Sciences.
\end{abstract}

Key concepts: teaching practices, immigrants, interculturality, History and Social Sciences 


\section{Introducción}

En los últimos 25 años se ha cuadruplicado el número de inmigrantes en Chile y actualmente representan el 6,6\% de la población nacional (Instituto Nacional de Estadística [INE] y Departamento de Extranjería y Migración [DEM], 2018). Estas cifras se reflejan en el sistema educacional: entre 2005 y 2016 la matrícula de estudiantes inmigrantes aumentó seis veces, lo que representó el 1,7\% de la matrícula nacional (Joiko y Vásquez, 2016). Solo en el transcurso de 2015 a 2016 la matrícula extranjera aumentó un 99,5\% (Silva y Ballesteros, 2017), llegando en 2018 a significar el 3,2\% de la matrícula nacional (MINEDUC, 2018).

El incremento de la inmigración ha significado el desafío de integrar a niños, niñas y adolescentes (NNA) migrantes al sistema educacional y, también, a los beneficios derivados de la asistencia material y programas de apoyo. Desde octubre de 2016 se encuentra funcionando la "Mesa de Coordinación Migrante" (MCM), que tiene tres áreas programáticas, una de las cuales es el "fortalecimiento educativo" y cuyo objetivo es elaborar políticas públicas para abordar el tema de la educación de NNA migrantes, en consonancia con los principios de inclusión e interculturalidad, ya consagrados en la legislación educacional chilena (República de Chile, 2009 y 2015).

Entre las políticas públicas más importantes para la inserción de los NNA migrantes en el sistema educativo, fue crucial, en un primer momento, la creación del Identificador Provisorio Escolar (IPE) para estudiantes migrantes (en reemplazo del RUT 100) y, más recientemente, la creación de una visa temporaria especial de residencia totalmente gratuita para los NNA extranjeros, con la finalidad de regularizar su situación legal, permitirles la obtención de su cédula de identidad y el acceso a los mismos beneficios educacionales y prestaciones de salud disponibles para NNA chilenos (DEM, 2017).

La integración de los NNA migrantes trajo consigo el reto de educar en la misma escuela a estudiantes que poseen referentes culturales distintos, pero que necesitan comprenderse mutuamente 
para vivir juntos y elaborar proyectos de sociedad compartidos (MINEDUC, 2017; Poblete, 2018). Este reto es posible de abordar mediante una educación con enfoque intercultural, pero, aunque ello esté declarado en las políticas educacionales chilenas, existen realidades que la obstaculizan, tales como el racismo y la discriminación al interior de las escuelas (Riedemann y Stefoni, 2015; Tijoux, 2013). Otro de los obstáculos es la ausencia de orientaciones conceptuales, técnicas y pedagógicas. Ello implica que las escuelas no cuentan con ayuda para aprender qué significa la educación con enfoque intercultural, analizar su propio escenario aplicando herramientas conceptuales de dicho enfoque y modificar sus dinámicas institucionales y sus prácticas pedagógicas para implementarlo. De este modo, la nueva tarea se suma a la larga lista de exigencias tradicionales, tales como la presión por cubrir un curriculum extenso en poco tiempo (Stefoni, Stang y Riedemann, 2016), y rendir bien en las mediciones estandarizadas, entre otras, en lugar de representar una oportunidad para replantear los propósitos de la institución y mejorar el proceso educativo para los estudiantes en su conjunto.

Uno de los actores cruciales en la construcción de escuelas interculturales son los y las docentes. En el ámbito académico, los principales desafíos enfrentados por los profesores se relacionan con el curriculum, las estrategias de enseñanza y la nivelación de estudios de los estudiantes extranjeros (Poblete y Galaz, 2017; Servicio Jesuita Migrante [SJM], 2018) $)^{5}$. En cuanto al curriculum, los profesores consideran que no les ofrece la pluralidad de contenidos y estrategias de enseñanza que ellos perciben como necesarios para atender adecuadamente la diversidad cultural (Poblete y Galaz, 2016), lo que acarrea la falta de identificación de los alumnos migrantes con lo enseñado en la escuela y se transforma en otro de los factores que dificulta su aprendizaje (Joiko y Vásquez, 2016; Fundación Superación de la Pobreza [FSP] y Instituto de Estudios Municipales

5 En 2017, el servicio Jesuita Migrante (SJM) llevó a cabo la aplicación piloto del "Programa Migración y Escuela" en cuatro colegios, con estudiantado migrante de las comunas de Arica, Antofagasta, Estación Central y Santiago. Las comunidades educativas de esos establecimientos levantaron desafíos y propuestas en distintos ámbitos del quehacer escolar (entre ellos, el ámbito académico) para construir escuelas interculturales. 
[ICHEM], 2016). En el área de Historia, Geografía y Ciencias Sociales (H, G y CCSS), este problema es particularmente sensible, puesto que muchos de los contenidos del curriculum se relacionan con conflictos bélicos entre Chile y países vecinos, de los cuales provienen muchos de los alumnos migrantes. Un ejemplo de esto lo representa la llamada "Guerra del Pacífico". Además, independientemente de los contenidos específicos prescritos, el curriculum del área se encuentra atravesado en todos los niveles de enseñanza por conceptos tales como "nación", "país", "identidad", entre otros, cuyo aprendizaje es crucial en la relación entre personas de diferentes nacionalidades y culturas.

De ello se desprende la necesidad de realizar adecuaciones a los contenidos curriculares, que consideren los referentes culturales del estudiantado, es decir, contextualizar el curriculum escolar, además de implementar estrategias didácticas para enseñar a estudiantes culturalmente diversos y adecuar los procesos de enseñanzaaprendizaje para lograr que todos y todas aprendan (Poblete y Galaz, 2016; SJM, 2018).

Consecuentemente con la realidad descrita, investigaciones (Martínez, 2018) han recomendado fortalecer las competencias de desarrollo curricular de las escuelas, de modo de permitirles contextualizar y ajustar las bases curriculares y programas de estudio, haciéndose cargo de los requerimientos específicos de cada una de las comunidades educativas (Mondaca, Gajardo y Sánchez, 2014; FSP \& ICHEM, 2016).

No obstante, los docentes, al igual que las instituciones escolares, no reciben orientaciones conceptuales ni didácticas para implementar una enseñanza con enfoque intercultural, razón por la que muchos de ellos han tenido que generar respuestas intuitivas a los desafíos de trabajar en escuelas con alumnos migrantes. En la asignatura H, G y CCSS muchos docentes han desarrollado adecuaciones curriculares consistentes, por ejemplo, en seleccionar elementos históricos y culturales de la cultura de origen de sus estudiantes migrantes, y enseñarlos conjuntamente con los contenidos obligatorios del curriculum chileno (Joiko y Vásquez, 2016; FSP e ICHEM, 2016). 
Los docentes que diseñan e implementan este tipo de adecuaciones curriculares todavía no han tenido la oportunidad de aprender herramientas conceptuales propias de un enfoque intercultural; sin embargo, son capaces de llevar a la práctica acciones que podríamos asociar con algunos principios y lógicas de dicho enfoque. En el ejemplo referido, los docentes están poniendo a disposición de todos sus estudiantes, contenidos históricos propios de cada una de sus diferentes culturas de origen, en un momento y espacio que, teóricamente, estaba destinado al aprendizaje de contenidos históricos propios de una de las culturas de los presentes en la sala de clases, la cultura de la sociedad de llegada. Esta situación implicó que el docente propiciara una situación en la que sus estudiantes tuvieran la oportunidad de conocer elementos culturales de sus compañeros y comenzaran a dialogar acerca de estos.

Hasta el momento se dispone de un importante conocimiento sociológico respecto de la escuela chilena como espacio de interacciones entre nativos y migrantes (Poblete, 2018; Poblete y Galaz, 2017; Tijoux, 2013; Riedemann y Stefoni, 2015; Stefoni et al., 2016), pero este espacio no ha sido explorado desde el punto de vista de la investigación pedagógica disciplinar, en este caso enfocada en el área de la H, G y CCSS.

Debido a que los docentes no cuentan con una fundamentación conceptual, probablemente estas prácticas emergentes sean difusas, poco sistemáticas e incompletas desde el punto de vista de lo que la literatura experta ha definido como una "enseñanza con enfoque intercultural". Por ejemplo, es posible que los profesores intencionen el diálogo entre distintas posiciones y saberes culturales, pero no propicien "la construcción conjunta de posibilidades y estrategias" o "una reflexión crítica sobre aquello que se identifica como las formas culturales propias" (Stefoni et al., 2016, p. 160). No obstante, prácticas docentes como la descrita emergen desde contextos escolares reales y, por lo mismo, tienen la virtud de haber sido pensadas y diseñadas para ser viables en esos contextos escolares específicos. Debido a ello, investigarlas y sistematizarlas permite contar con una base auténtica de conocimiento, que alimente el diseño de futuros programas de ayuda y orientación didáctica para 
la implementación de la enseñanza con enfoque intercultural en el área de H, G y CCSS.

El objetivo de este trabajo es caracterizar prácticas docentes, que incluyen aspectos históricos y culturales de los alumnos inmigrantes, en la enseñanza de los contenidos mínimos del curriculum del área de Historia, Geografía y Ciencias Sociales, con el propósito de describir estrategias de interculturalidad surgidas al interior de escuelas con alta matrícula inmigrante.

A continuación se presentará el marco teórico y la metodología que orientó esta investigación. Posteriormente, el análisis de los resultados organizado en tres secciones: en la primera de ellas se explicarán las características de las prácticas docentes de los profesores observados, en términos de las estrategias de enseñanza utilizadas, las formas de implementación de dichas estrategias y las explicaciones de los docentes respecto de sus propias prácticas. En la segunda sección del análisis de resultados nos referiremos a los contenidos históricos y los aspectos culturales de los alumnos inmigrantes que los profesores recuperan e incorporan a las clases de H, G y CCSS, y en la tercera abordaremos las prácticas docentes que se apartan de un enfoque intercultural. En la parte final se proponen algunas conclusiones derivadas del análisis realizado.

\section{Marco teórico}

El marco de referencia del estudio se organiza en cuatro secciones. En la primera se identifican los principales elementos teóricos de la investigación, en la segunda sección se aborda el concepto de "interculturalidad", en la tercera se muestran algunas investigaciones referidas a la educación intercultural y la enseñanza de la H, G y CCSS y, por último, en la cuarta sección se examinan investigaciones realizadas en escuelas chilenas con alumnos migrantes.

\subsection{Principales elementos teóricos de la investigación}

A continuación, nos referiremos a los cuatro principales elementos teóricos involucrados en esta propuesta de investigación: 


\section{(1) Interculturalidad}

Entenderemos por interculturalidad "una situación, proceso o proyecto que se produce en el espacio entre dos distintas formas de producción cultural" (Novaro, 2006, citado en Stefoni et al., 2016, p. 159). La interculturalidad

supone la posibilidad de un encuentro igualitario entre grupos diversos, mediante el diálogo entre distintas posiciones y saberes, y la construcción conjunta de posibilidades y estrategias (...) pero además implicaría una reflexión crítica sobre aquello que se identifica como las formas culturales propias, lo que permitiría entender su carácter relativo e histórico. (Stefoni et al., 2016, p. 160)

\section{(2) Las prácticas docentes}

En las prácticas docentes del área de H, G y CCSS se puede observar tres elementos: (1) historia disciplinar y ciencias sociales; (2) saber seleccionado por las sociedades para ser transmitido (documentos curriculares oficiales) y (3) los usos, reelaboraciones, relecturas que los docentes hacen del saber seleccionado y las evaluaciones (Gregorini, 2015).

Esta investigación estudió el tercer elemento de las prácticas docentes (usos, reelaboraciones y relecturas que los docentes hacen del saber seleccionado).

\section{(3) Historia y Ciencias Sociales}

Tanto la Historia como las Ciencias Sociales son disciplinas que permiten explicar los procesos, relaciones y fenómenos que tienen lugar dentro de la sociedad. Dos de sus principales conceptos son el "tiempo" y el "espacio", los cuales entregan categorías para analizar la realidad social presente y pasada, tomar conciencia de participar de un pasado compartido con otros (colectivo social), empatizar con los demás y, también, de escoger formas de participar en el presente y el futuro de la sociedad. Por ello, la Historia y las Ciencias Sociales han sido relevadas como áreas de estudio en el curriculum escolar nacional (MINEDUC, 2009, 2012, 2016). 
(4) Curriculum de H, Gy CCSS

El curriculum chileno de H, G y CCSS propicia la posibilidad de concretar una educación antirracista y tendiente a un enfoque intercultural (MINEDUC, 2012, 2016). De acuerdo con Riedemann y Stefoni (2015), sería necesario potenciar en el curriculum la enseñanza de algunos contenidos específicos y otros más teóricos y conceptuales. En el caso de los contenidos específicos, puede mencionarse la conquista de América, las causas de la migración a nivel global y la formación de la sociedad colonial americana bajo la influencia de los criterios europeos del color de la piel. Entre los contenidos teóricos se cuentan los conceptos de "racismo biológico" y "racismo cultural".

Desde nuestra revisión preliminar del curriculum, apreciamos que existen muchos contenidos oportunos para trabajar la interculturalidad, específicamente en los Organizadores Temáticos (OT) de las Bases Curriculares (BBCC) de $7^{\circ}$ básico a $2^{\circ}$ medio. Algunos de estos son los siguientes: (1) Ejemplos de convivencia: "analizar ejemplos de relaciones de influencia y convivencia entre el mundo europeo, el bizantino y el islámico durante la Edad Media" (7ºásico, OT11); (2) todos los relativos a formación ciudadana, en todos los niveles de enseñanza; (3) contenidos controversiales que involucran a actores chilenos y de las nacionalidades de los alumnos migrantes (por ejemplo, Guerra del Pacífico, $1^{\circ}$ medio, OT15); (4) los procesos históricos que muestran rupturas violentas de la convivencia (por ejemplo, Segunda Guerra Mundial y Holocausto judío), y (5) los procesos históricos simultáneos en distintos países de América Latina, los cuales permitirían abordar la historia de Chile, sus vecinos y otros países latinoamericanos desde el punto de vista de sus similitudes y los elementos históricos que los unen (por ejemplo, Independencia de las colonias hispanoamericanas (8básico, OT16) (MINEDUC, 2016).

\subsection{El concepto de "interculturalidad"}

Para aproximarse al concepto de "interculturalidad" es necesario definir qué entendemos por "cultura". Para efectos de esta investigación, puede definirse como "un sistema de conceptos, convicciones, actitudes y valores que se expresa en la forma de actuar 
y en las producciones materiales e inmateriales de las personas" (Maleztzke, 1996, citado en Garcés, Riedemann, Naudon y Palma, 2017, p. 40).

Intuitivamente, puede inferirse que el concepto de "interculturalidad" refiere a la relación entre culturas distintas. En efecto, durante la segunda mitad del siglo XX, en el contexto de la descolonización europea, proliferan los conceptos para denominar las relaciones culturales sin sesgo de dominación entre culturas, buscando reflejar relaciones más simétricas en términos de la valoración o legitimidad de culturas diferentes.

Así, en el mundo anglosajón comienza a hablarse de "multiculturalidad". Este término surgió en los años 60 a partir de un grupo de movimientos sociales que abogaban por la valoración de la diversidad, fenómeno que llegó a denominarse "movimiento multiculturalista" (Dietz, 2012, p. 19). La idea básica que compartía este conjunto de movimientos sociales desafiaba la noción clásica de que todos los seres humanos somos iguales y, por tanto, sujetos de los mismos derechos y obligaciones. En cambio, se introdujo la noción de que los seres humanos somos distintos y diversos, y de que no necesariamente es justo ofrecer el mismo trato a quienes son distintos. Bajo esta premisa, este movimiento perseguía el reconocimiento de la diversidad en la sociedad y el desarrollo de espacios en los que ésta pudiera expresarse (Garcés et al., 2017, p. 38). Estas ideas tuvieron como correlato político el surgimiento de la "acción afirmativa" o "discriminación positiva", una forma de orientar políticas públicas hacia grupos sociales históricamente oprimidos o discriminados, para obtener resultados o permitir su participación de manera equitativa.

En Europa continental, en cambio, los fenómenos migratorios poscoloniales dieron origen a la noción de "interculturalidad", que enfatiza la responsabilidad de las mayorías sociales de acoger la diversidad y enfrentar sus desafíos (PRIEM y FSP, 2017, p. 16). Esta noción se extendió a América Latina, aunque con énfasis particular en la relación entre el Estado y los pueblos originarios, bajo la nominación de "interculturalidad bilingüe" (Garcés et al., 2017, p. 38). Por tanto, el significado del concepto de "interculturalidad" en 
nuestro continente y en nuestro país ha sido limitado y circunscrito a la relación entre Estado y pueblos indígenas originarios. Esta situación ha llevado a reconocer la diversidad cultural y a otorgar derechos específicos a ciertas agrupaciones, pero solo dentro de los límites de la política institucional establecida, sin cuestionar de manera eficaz las estructuras y dispositivos que mantienen la desigualdad social y cultural (Walsh, 2009; Diez, 2004).

Desde estas problemáticas, en Latinoamérica emerge el enfoque intercultural orientado hacia una perspectiva crítica, que cuestiona la "estructura y/o matriz colonial de poder racializado y jerarquizado, que posiciona a los blancos y 'blanqueados' en la cima y los pueblos indígenas y afrodescendientes en los peldaños inferiores" (Walsh, 2009 , p. 4). De esta forma, la interculturalidad puede definirse como "una situación, proceso o proyecto que se produce en el espacio entre dos distintas formas de producción cultural" (Novaro, 2006, citado en Stefoni et al., 2016, p. 159), y que, "a través de la comunicación, busca transformar las estructuras de poder que inferiorizan, deshumanizan y racializan" (Garcés et al., 2017, pág. 41). Dicho de otro modo, la interculturalidad crítica se orienta en la co-construcción de un proyecto político, social, ético y epistemológico, que cuestiona y reconoce que las relaciones entre culturas se producen en un contexto social particular, marcado por relaciones de poder, dominación y jerarquías (Walsh, 2009; Diez, 2004).

La interculturalidad busca generar relaciones de simetría en el contacto entre culturas (Tubino, 2005, citado en Garcés et al., 2017, p. 40) reconociendo la importancia del conflicto, en cuanto permite develar las estructuras de poder funcionales a la desigualdad. Más específicamente, la interculturalidad

supone la posibilidad de un encuentro igualitario entre grupos diversos, mediante el diálogo entre distintas posiciones y saberes y la construcción conjunta de posibilidades y estrategias (...) pero además implicaría una reflexión crítica sobre aquello que se identifica como las formas culturales propias, lo que permitiría entender su carácter relativo e histórico. (Stefoni et al., 2016) 
Tradicionalmente se ha señalado al ámbito educativo como el espacio para la resolución de conflictos relacionados con las diversidad social y cultural. No obstante, es fundamental reconocer que las escuelas y las instituciones encargadas de los proyectos educativos se desarrollan en un contexto particular, por ende, el desarrollo de una educación intercultural no puede darse de manera aislada, cuando estructuralmente hay condiciones sumamente desiguales para ciertos grupos de la población (Diez, 2004). Por ello, la interculturalidad, desde un enfoque crítico, se posiciona como un proyecto político que debe ser parte de diversos espacios, ya que "los proyectos educativos no pueden suplir la ausencia de políticas interculturales" (Díaz, 2001, citado en Diez, 2004, p. 193).

En un contexto de aumento de inmigrantes en Chile en las últimas décadas, la diversidad cultural ha presentado nuevos desafíos a los establecimientos educacionales y al sistema en su conjunto. La noción europea de "interculturalidad" descarta los programas focalizados como el chileno, al plantear objetivos más ambiciosos, como por ejemplo lograr, mediante la educación intercultural,

una educación de calidad para todos y, en consecuencia, más eficaz (en el sentido de cumplir lo que prescriben las leyes) y más justa (en la línea de hacer frente a todas las desigualdades que impidan o condicionen una verdadera igualdad de oportunidades educativas y sociales) (Besalú, n.d., citado en PRIEM y FSP, 2017).

2.3. Investigaciones acerca de la educación intercultural y la enseñanza de la H, G y CCSS

Diversas investigaciones destacan que las condiciones sociopolíticas y culturales en las que se desarrollan los procesos de escolarización en distintos Estados-nación han cambiado como consecuencia de los procesos de modernización, globalización y migración (Hajisoteriou, Karousiou \& Angelides, 2018; Hornberg, 2002; Klein, 2010). En ese contexto, los sistemas educativos han ido avanzando hacia el desarrollo y fomento de prácticas educativas interculturales en aulas primarias y secundarias. Atendiendo a ello, se han multiplicado los estudios acerca del enfoque intercultural en la enseñanza de la H, G 
y CCSS. Algunos de los principales temas de estas investigaciones dicen relación con el desarrollo de la empatía intercultural en la sala de clases y la necesidad de abordar las perspectivas históricas y culturales de los estudiantes inmigrantes en la asignatura de H, G y CCSS (Zembylas \& Kambani, 2012; Hawley, Crowe \& Mooney, 2016; Ho, McAcoy, Hess \& Gibbs, 2017; Solhaug \& Osler, 2018, entre muchos otros).

Investigaciones internacionales recientes se han abocado al estudio de la forma en que la asignatura de H, G y CCSS puede contribuir al desarrollo de competencias interculturales y cuáles serían las implicancias de ello en cuanto a contenidos y métodos de enseñanza (Nordgren, 2017).

Nordgren y Johansson (2015) realizaron un cruce entre las dimensiones relacionadas con la competencia intercultural y las habilidades asociadas a la conciencia histórica. Como resultado, elaboraron una matriz que muestra cómo los conceptos históricos e interculturales se pueden enriquecer mutuamente (tabla 1). La matriz se estructura en torno a tres dimensiones de la competencia intercultural: (1) conocimiento de procesos sociales y culturales; (2) habilidades para interpretar representaciones de otras culturas y para conectar dichas representaciones con su propia cultura, y (3) habilidad para descentrarse. La primera dimensión se relaciona con la capacidad de experimentar el tiempo y los eventos históricos que transcurren en este; la segunda con la de interpretar el sentido del pasado en forma de relato histórico, en tanto que la tercera dimensión se refiere, por un lado, a la comprensión de que los propios valores, creencias y comportamientos no son los únicos posibles y, por otro, a la aplicación de las dos habilidades anteriores para dar sentido a situaciones contemporáneas. 
Tabla 1.

Matriz de aprendizaje histórico intercultural (Nordgren y Johansson, 2015).

\begin{tabular}{ll}
\hline \multicolumn{1}{c}{ Dimensiones competencia intercultural } & \multicolumn{1}{c}{$\begin{array}{c}\text { Habilidades asociadas a la conciencia } \\
\text { histórica }\end{array}$} \\
\hline $\begin{array}{l}\text { Conocimiento de procesos sociales } \\
\text { y culturales: incluye conocimiento } \\
\text { sobre fenómenos culturales, sociales } \\
\text { e históricos, tales como diferencias } \\
\text { culturales dentro de y fuera del Estado- } \\
\text { nación. }\end{array}$ & $\begin{array}{l}\text { Habilidad de experienciar: referida a la } \\
\text { sensibilidad respecto del paso del tiempo } \\
\text { en nuestro entorno, considerando el } \\
\text { conocimiento de eventos y conceptos } \\
\text { históricos. }\end{array}$ \\
\hline $\begin{array}{l}\text { Habilidad para interpretar } \\
\text { representaciones de otras culturas y para } \\
\text { conectar dichas representaciones con } \\
\text { su propia cultura: incluye habilidades } \\
\text { para tomar otras posiciones, interpretar } \\
\text { representaciones desconocidas y mediar } \\
\text { entre perspectivas diversas. }\end{array}$ & $\begin{array}{l}\text { Habilidad de interpretar: orientada a } \\
\text { relato histórico. Esto implica comprender } \\
\text { el significado o la importancia de los } \\
\text { cambio. las causas de un proceso de }\end{array}$ \\
\hline $\begin{array}{l}\text { Habilidad para descentrarse: comprender } \\
\text { que los propios valores, creencias y }\end{array}$ & $\begin{array}{l}\text { Habilidad de orientar: refiere al acto de } \\
\text { utilizar las habilidades de experienciar } \\
\text { comportamientos no son los únicos } \\
\text { posibles. }\end{array}$ \\
$\begin{array}{l}\text { e interpretar la historia con el propósito } \\
\text { contergar sentido a situaciones }\end{array}$ \\
\hline
\end{tabular}

Elaboración propia con base en la traducción libre de "Matrix of intercultural historical learning". Nordgren, K. \& Johansson, M. (2015). Intercultural historical and history education. Journal of Curriculum Studies, 47(1), 10.

En consecuencia, es posible afirmar que la literatura internacional coincide en señalar que H, G y CCSS es un área del curriculum escolar que otorga la oportunidad para comprender la sociedad en la que se vive y desarrollar competencias interculturales (Angvik \& Von Borries, 1997; Carretero \& Voss, 1994; Barton \& Levstik, 2004).

\subsection{Investigaciones en escuelas chilenas con alumnos migrantes}

En Latinoamérica, las investigaciones acerca de educación intercultural en el ámbito de la enseñanza se han centrado mayoritariamente en el ámbito de lengua y cultura indígena. Para el caso chileno, hasta el momento, no se dispone de estudios que se aboquen, específicamente, a la enseñanza con enfoque intercultural de la H, G y CCSS en contextos de escuelas con alumnos migrantes. Aunque, desde el punto de vista del estudio de la discriminación y el racismo, varias investigaciones se han adentrado en el mencionado contexto escolar. 
Estos estudios nos permiten efectuar la siguiente sistematización de las necesidades de las escuelas respecto de las posibilidades de implementar una educación intercultural: (a) instancias de reflexión de la comunidad educativa acerca de la discriminación y el racismo (Tijoux, 2013); (b) instancias de aprendizaje de los docentes de marcos conceptuales acerca de la interculturalidad y de educación antirracista (Poblete, 2018; PRIEM y FSP, 2017; Riedemann y Stefoni, 2015), y (c) modelos de implementación curricular que consideren las culturas de origen de los estudiantes, a partir de las cuales se genere un diálogo y mutua comprensión (Stefoni et al., 2016). Asimismo, las investigaciones ponen de manifiesto que, concomitantemente con los obstáculos y las necesidades, tienen lugar prácticas que docentes, directivos y otros miembros ya han implementado para enfrentar los desafíos de educar a un alumnado con distintos referentes culturales (Stefoni et al., 2016; FSP \& ICHEM, 2016).

De este modo, a pesar de no tratarse del tema principal de los mencionados estudios, estos incluyen testimonios de profesores que refieren lo poco significativo que resulta para los alumnos inmigrantes el estudio de algunos elementos del curriculum chileno. Un ejemplo de ello son los héroes de la historia chilena y cómo el hecho de incluir también a los héroes de la historia de los inmigrantes hace que tanto niños chilenos como migrantes se interesen más en la asignatura de H, G y CCSS, y entablen una conversación (Joiko y Vásquez, 2016). Por otro lado, desde el MINEDUC, centros de estudios especializados y también fundaciones, trabajos de exploración en establecimientos educacionales chilenos han dado lugar a valiosos documentos de orientaciones y guías pedagógicas para una educación intercultural (PRIEM y FSP, 2017; MINEDUC, 2017; FSP \& ICHEM, 2016). Dichos trabajos dan cuenta de adaptaciones curriculares realizadas por profesores de Historia para facilitar el aprendizaje de los niños inmigrantes. Entre estas adaptaciones destaca el tratamiento de procesos de la historia de Chile en paralelo con la historia de otros países latinoamericanos, específicamente procesos independentistas del siglo XIX, flujos migratorios campo-ciudad de fines del siglo XIX y comienzos de XX, entre otros (FSP \& ICHEM, 2016). 
En nuestro país, el trabajo de Martínez (2018) analizó los elementos socioculturales del sistema escolar en Chile y España, considerando aspectos tales como los marcos normativos, las políticas públicas en migración y educación, las relaciones al interior de las escuelas y las relaciones familia-escuela. Para el caso de Chile, en el ámbito de los contenidos curriculares, Martínez llama la atención acerca de la poca correspondencia entre la diversidad sociocultural de las escuelas y los contenidos curriculares, lo que, como ya mencionamos, desemboca en la falta de identificación de los alumnos migrantes con lo enseñado en la escuela. Dicho de otro modo, no existe una educación sensible a las pertenencias culturales de los alumnos y alumnas. Adicionalmente, Martínez coincide con los estudios de Poblete (2018); Poblete y Galaz (2017); Tijoux (2013); Riedemann y Stefoni (2015), y Stefoni et al. (2016), señalando que la falta de identificación con el curriculum tiene lugar de manera conjunta con la discriminación y racismo sufridos por los estudiantes migrantes dentro de las escuelas.

\section{Metodología}

El estudio se efectuó en la comuna de Santiago, debido a que es la comuna donde residen más personas extranjeras en Chile: 212.037 personas (INE \& DEM, 2018), y también, según el Sistema de Información General de Estudiantes para 2018, porque es la comuna con más estudiantes migrantes matriculados en todo Chile. Se consideró a las escuelas de dependencia administrativa municipal y particular subvencionada porque, entre ambas, atienden al 93,5\% de estudiantes inmigrantes (MINEDUC, 2018; Joiko y Vásquez, 2016). La población estuvo conformada por los profesores de la asignatura de H, G y CCSS que atienden los niveles educacionales de $7^{\circ}$ básico a $4^{\circ}$ medio en escuelas municipales y particulares subvencionadas con alto porcentaje de matrícula de alumnos migrantes, es decir, un porcentaje igual o superior a 15\% (Salas et al., 2017), ubicadas en la comuna de Santiago.

Se consideró a los niveles $7^{\circ}$ básico a $4^{\circ}$ medio porque, generalmente, son atendidos por profesores de Historia, de Educación Media, cuya especialización permite suponer que podrían tener 
mayor capacidad reflexiva acerca de las disciplinas del curriculum y, por ende, evaluar más posibilidades de relación de los contenidos del curriculum nacional con otros contenidos (en este caso, contenidos de la historia y la cultura de origen de los alumnos migrantes). La unidad de análisis fue conformada por profesores, por lo que no se distinguió entre las nacionalidades de los estudiantes.

Se optó por un diseño exploratorio, porque se buscó la aproximación a un fenómeno que, hasta el momento, no ha sido investigado. Se utilizó una metodología cualitativa con un enfoque Grounded Theory (Strauss y Corbin, 1990, citado en Sandín, 2003). La elección del enfoque se fundamenta en su capacidad de caracterizar y generar conocimiento de un fenómeno desde las prácticas (prácticas docentes) y desde los actores (docentes).

Se cauteló que la mitad de los profesores correspondieran a escuelas municipales y la otra mitad a escuelas particulares subvencionadas, y que entre los docentes estudiados hubiera paridad de género (dos hombres y dos mujeres). De este modo, se eligieron cuatro docentes de escuelas distintas.:

- Una docente de escuela municipal de niñas (Docente A).

- Un docente de escuela municipal mixta (Docente B).

- Un docente de colegio particular subvencionado mixto (Docente C).

- Una docente de colegio particular subvencionado de niñas (Docente D).

Las estrategias de recogida de información fueron la grabación audiovisual de clases y la entrevista con pauta semiestructurada (ver "Pauta de entrevista semiestructurada a docentes" en la sección anexos). Se realizaron 21 observaciones (cinco a los docentes A, B y $\mathrm{C}$, y seis a la docente D) e igual número de entrevistas. De lo anterior se obtuvo 31,5 horas de videograbación de clases y siete horas de grabación de entrevistas en audio.

Para el análisis de la información se aplicó el método de comparación constante y muestreo teórico de la Grounded Theory, codificando mediante el uso del software especializado ATLAS TI. Se aplicaron 12 códigos elaborados con base en las dimensiones y 
subdimensiones para la descripción y análisis de las prácticas docentes del área de Historia, Geografía y Ciencias Sociales planteadas por Gregorini, 2015 (tabla 2).

Tabla 2.

Dimensiones, subdimensiones, códigos y ejemplos para la descripción y análisis de las prácticas docentes del área de Historia, Geografía y Ciencias Sociales.

\begin{tabular}{|c|c|c|}
\hline Dimensiones & Subdimensiones & Códigos y ejemplos \\
\hline \multirow{3}{*}{ (3.1) Los usos } & Estrategias didácticas & $\begin{array}{l}\text { (C1) Expositiva. } \\
\text { (C2) Interactiva (por ejemplo, trabajo } \\
\text { grupal, disertaciones, etc.). }\end{array}$ \\
\hline & $\begin{array}{l}\text { Formas de } \\
\text { implementación de las } \\
\text { estrategias didácticas }\end{array}$ & $\begin{array}{l}\text { (C3) Conformación de grupos de } \\
\text { estudiantes utilizando criterios } \\
\text { aleatorios o (C4) intencionados. }\end{array}$ \\
\hline & Recursos didácticos & $\begin{array}{l}\text { (C5) Guías de estudio, (C6) } \\
\text { presentación de contenidos en Power } \\
\text { Point, (C7) cine, (C8) fotografía, etc. }\end{array}$ \\
\hline (3.2) Relecturas & Objetivo didáctico & $\begin{array}{l}\text { (C9) Descomposición de un } \\
\text { aprendizaje esperado (Programa de } \\
\text { Estudio) o (C10) elaboración propia. }\end{array}$ \\
\hline \multirow{2}{*}{$\begin{array}{l}\text { (3.3) Reelaboraciones } \\
\text { que los docentes } \\
\text { hacen del saber } \\
\text { seleccionado }\end{array}$} & \multirow{2}{*}{$\begin{array}{l}\text { (B) Tipos de aspectos } \\
\text { históricos y culturales } \\
\text { de los alumnos } \\
\text { inmigrantes que los } \\
\text { docentes incluyen } \\
\text { en la enseñanza } \\
\text { de los contenidos } \\
\text { obligatorios del } \\
\text { curriculum. }\end{array}$} & $\begin{array}{l}\text { (C11) Históricos, por ejemplo, } \\
\text { personajes, hitos históricos específicos } \\
\text { (Independencia, golpes de Estado), } \\
\text { procesos históricos o sociales, etc. }\end{array}$ \\
\hline & & $\begin{array}{l}\text { (C12) Culturales. } \\
\text { Ejemplos: costumbres, fiestas típicas, } \\
\text { comida, vestimenta, modos de ser, } \\
\text { valores, visiones acerca de la vida, etc. }\end{array}$ \\
\hline
\end{tabular}

Elaboración propia. Basada en Gregorini, V. (2015). La enseñanza de la historia. Una compleja relación entre norma y práctica. Revista Brasileira de Educación, 61, 457-478.

\section{Análisis de resultados ${ }^{6}$}

\subsection{Características de las prácticas docentes y explicaciones de los profesores}

Las estrategias utilizadas por los docentes pueden ser clasificadas en dos grandes grupos. (1) Combinación o integración de estrategias de enseñanza expositivas e interactivas, como una respuesta a

6 De acuerdo con la nomenclatura explicada en la sección "selección de los participantes", en lo sucesivo nos referiremos a los profesores como docente A, B, C y D, y a las clases observadas como C, indicando además el número de cada clase, es decir $\mathrm{Cl}, \mathrm{C} 2$, etc. De manera similar, las entrevistas serán referidas como E, indicando su correspondiente número. 
la necesidad de atender a estudiantes con diversos niveles de comprensión de los contenidos históricos. (2) Estrategias diseñadas por los profesores especialmente para la enseñanza de la H, G y CCSS en aulas conformadas por alumnos migrantes y no migrantes.

(1) Combinaciones originales de estrategias de enseñanza convencionales

Se observó una tendencia a la combinación de estrategias de enseñanza de tipo interactivo y expositivo dentro de la misma clase. Un ejemplo recurrente en las clases observadas fue la combinación de trabajo en grupo, seguido por puestas en común o plenarios guiados por el docente. Una de las formas de implementación fue la conformación de los grupos por el profesor, aplicando criterios de diferenciación de los estudiantes por nivel de desempeño en la asignatura. Respecto del "nivel de desempeño", resulta notable que los profesores no aludieron a las calificaciones como indicador de este, sino que al grado de "profundidad en la comprensión" de los contenidos de la asignatura demostrada por los estudiantes. Bajo esta forma de implementación, cada grupo queda conformado por integrantes de características relativamente homogéneas, pero el profesor incluye, de manera intencionada, a un estudiante de alto nivel de desempeño que guía el trabajo y ayuda a los demás integrantes. En uno de los casos estudiados, los estudiantes-guía eran niñas migrantes:

hay un grupo de niñas específicamente que son las que tienen mayores capacidades; coincidentemente, este grupo de niñas también son las niñas venezolanas iya?, por lo tanto, como estas niñas tienen esta capacidad de liderazgo y de fácil comprensión, tampoco son egoístas también cuando tienen algo y conocen algo tratan de compartirlo, y son líderes totalmente positivos dentro del curso, por lo tanto, una vez que ellas entienden algo lo comparten con los compañeros y tratan de llevar a los compañeros a avanzar en el aprendizaje. (DB, E1)

El profesor asigna a cada uno de los grupos actividades referidas al mismo contenido curricular, pero con distinto grado de dificultad, de manera que resulten abordables y que, posteriormente, todos los grupos puedan aportar en los momentos de puesta en común o plenario. 
Los docentes estudiados explican la aplicación de combinaciones de estrategias como una respuesta a la necesidad de atender a estudiantes diversos:

Mira, principalmente porque esta escuela es una escuela que tiene proyecto de integración, y así como tenemos niños extranjeros también tenemos niños con necesidades educativas especiales, entonces tenemos que apuntar principalmente a lo que es kinestésico, a lo que es visual y lo que puede ser auditivo iya?, entonces, con lo kinestésico, que los niños se pusieran de pie y fueran a ubicar algo era un apoyo, y también, por el otro lado, lo visual y lo kinestésico, al tener que elaborar el mapa, al tener que trabajar todo eso, y además me he encontrado en ocasiones en que hay niños que, sobre todo extranjeros, que traen cierto vacío en cuanto a contenido, si tú te fijas habian niños que decían "no, esto lo vimos en cuarto básico", pero hay otros niños que para ellos es totalmente nuevo esta materia, entonces por eso tenía que tomar, tomé la decisión de hacerlo desde distintas estrategias. (DB, E1)

Tal como se señala en este fragmento de entrevista, la diversidad que los docentes enfrentan se refiere no solo al ámbito cultural. En las salas observadas fue común encontrar niños con distintos tipos de necesidades educativas especiales, tales como, hipoacusia, espectro autista y deficiencia intelectual leve o moderada, entre otras. Ello configura un escenario de alta complejidad, ante el cual los docentes responden aplicando estrategias de distinta naturaleza. En este artículo reportamos una de las combinaciones más recurrentes, pero también fue posible apreciar otras "versiones". En nuestra opinión, cada una de estas corresponde a síntesis más o menos originales y su originalidad radica en el hecho de que cada una de ellas responde a contextos similares, pero no idénticos, y a la especificidad de dichos contextos, por ello algunos elementos (recursos didácticos, formas de implementación, tiempos, etc.) son enfatizados por sobre otros.

(2) Estrategias diseñadas especialmente para la enseñanza de la Historia, G y CCSS en aulas conformadas por alumnos migrantes y no migrantes

Se encontró principalmente dos tipos de estrategias: la comparación entre las características del mismo hecho, proceso histórico o tema 
en Chile y en los países de origen de los alumnos inmigrantes, y el uso de analogías entre las experiencias de los alumnos inmigrantes y los contenidos curriculares obligatorios de la asignatura de $\mathrm{H}, \mathrm{G}$ y CCSS. Fundamentalmente, estas estrategias se presentan en forma de exposiciones de los docentes y clases dialogadas. A continuación, mostramos referencias a ambos tipos de estrategias observadas:

- La comparación entre las características del mismo hecho, proceso histórico o tema en Chile y en los países de origen de los alumnos inmigrantes

... el año pasado era el tema de la organización política de Chile, ¿ya?, en sexto básico, entonces, también hacíamos comparaciones respecto a cómo era la forma de organización política de Chile y cómo era la de Venezuela, que, desde mi punto de vista, me llevé una sorpresa. Me di cuenta de que los niños de Venezuela, obviamente por el contexto histórico que tienen, sabian mucho acerca de conceptos como "Constitución", como "República", como "Democracia", (...) a diferencia del niño chileno, que (...) no le toma mucho el peso, o sea, si tú le hablas de República, si tú le hablas de, de Constitución, por ejemplo, para los chilenos es algo abstracto, algo lejano, en cambio para, para el niño venezolano es sumamente potente, está muy empoderado en ese tema, y que también es un aspecto muy positivo porque le permitió a los niños chilenos tomar conciencia de esos elementos. (DB, E1)

En este caso podemos apreciar cómo el docente se apoya en los conocimientos previos de un grupo de estudiantes inmigrantes (venezolanos) para abordar un contenido que, de acuerdo con el curriculum nacional, debe ser apropiado por parte de los estudiantes nacionales y extranjeros, acto que involucra una valoración, en el espacio de aula, del acervo cultural extranjero. Similar caso es el de una de las docentes que, al examinar el fenómeno de la esclavitud en la época colonial, recoge las palabras de una de las estudiantes:

Es importante lo que usted decía Alejandra [nombre ficticio] porque nosotros pensamos que en Chile no hubo, pero sí hubo esclavitud (...) porque siempre se tiende a pensar que la mayor población esclava fue hacia solamente Brasil, pero también como dice usted a los países tropicales. (DA, C1) 
- El uso de analogías entre las experiencias de los alumnos inmigrantes y los contenidos curriculares obligatorios de la asignatura de H, G y CCSS

Profesora: necesito preguntarles algo. María [nombre ficticio], con la llegada suya de Venezuela a Chile ¿cómo la impacto eso a usted? Alumna migrante: ¿cómo así?

Profesora: ¿generó un cambio en su vida diaria? En su alimentación, por ejemplo, en su vestimenta, en el champú no sé, yo nunca he vivido, yo solo he ido de vacaciones a otro país, pero yo no he vivido en otro país. ¿Cómo ha sido su experiencia?

Alumna migrante: buena, en la alimentación bastante.

Profesora: ¿por qué?

Alumna migrante: porque allá tampoco había mucha comida.

Profesora: no se encontraba la comida iya?

Alumna migrante: y acá sí, acá por lo menos se ve bastante, uno compra lo necesario y come, más que todo, lo que no había antes allá. Profesora: pero los alimentos, ison lo mismo? O, a pesar de que sean similares, algo cambia, el sabor quizás, no sé...

(...)

Alumna migrante: Miss, sabe que el agua...

Profesora: ien qué sentido?

Alumnas migrantes: jel cloro! (mencionan distintas alumnas migrantes al mismo tiempo).

Profesora: el cloro. Asqueroso ¿cierto?

Alumna migrante: sí, en Venezuela el agua no tiene cloro, es agua nada más así...

Profesora: ya, entonces para ustedes también hubo un impacto (...).

Entonces la idea, que al igual como usted pudo escuchar y usted misma puede recordar los cambios que ha tenido, podamos leer, saber y precisar los cambios que vivió América (abre la puerta de la sala de clases porque hay una alumna afuera) porque para usted, Luisa [alumna chilena, nombre ficticio], ¿qué cambios ha tenido tener compañeras extranjeras? ¿En la comida? ¿En los olores?

Alumna (Luisa): en los gritos.

Profesora: en los gritos, en la cultura.

(Todas las alumnas se ríen). (DA:C6) 
La construcción de la analogía, mediada por la profesora, a partir de las experiencias estudiantiles, resulta especialmente interesante, con miras a proyectar prácticas de enseñanza de la historia con enfoque intercultural, en al menos dos sentidos. En primer lugar, legitima que la discusión en el aula incorpore la experiencia vital de una estudiante de origen venezolano en relación con los cambios experimentados en su proceso de inserción a Chile. Y, en segundo lugar, procura que la analogía planteada aborde, en igualdad de condiciones, las experiencias de estudiantes chilenas. No obstante, resulta llamativo el hecho de que, pese a que una estudiante señala que en su país de origen vivió una situación de carencia de alimentos, la profesora no incorpore esta nueva información como una herramienta adicional al análisis. Ello podría reflejar algún grado de rigidez en la implementación de la estrategia de la analogía.

\subsection{Los contenidos de la historia y la cultura de los alumnos inmigrantes recuperados en las clases}

En las estrategias diseñadas especialmente para la enseñanza de la H, $G$ y CCSS en aulas con alumnos de distinto país de origen (comparación y analogías), el denominador común es la recuperación de elementos relacionados con la historia y la cultura de las sociedades de origen de los alumnos inmigrantes. Estos elementos corresponden a (1) contenidos histórico-disciplinares (aprendidos en la escuela y fuera de ella) y (2) elementos culturales relacionados con las costumbres, valores y experiencias en general que los alumnos migrantes "traen" desde sus países, así como también las situaciones vividas en Chile, pero que han sido derivadas de su condición de inmigrantes (trámites legales, confusiones por uso de modismos, entre otras).

Un ejemplo de contenidos histórico-disciplinares aprendidos en la escuela del país de origen son los conceptos de "Constitución", "república" y "democracia" de los niños venezolanos referidos más arriba (DB, E1). A juicio del profesor, el grado de apropiación del concepto por parte de los alumnos fue reforzado por el contexto histórico-social previo a su arribo a Chile. 

GEOGRAFÍA Y CIENCIAS SOCIALES, EN ESCUELAS CON ALTO PORCENTAJE DE ALUMNOS MIGRANTES DE LA COMUNA DE SANTIAGO - L. Valledor, L. Garcés, P. Whipple

En cuanto a las percepciones, un ejemplo que apareció en más de una oportunidad en las clases observadas se relaciona con el clima en la zona central de Chile: "ustedes conocen otro lugar de clima mediterráneo, ¿cómo es el clima en Venezuela?" (DB, C2). En este caso, el profesor acude a la experiencia de los estudiantes caraqueños con el clima templado (que es solo uno de los climas de Venezuela).

Las explicaciones de los docentes respecto de este tipo de prácticas aluden a propósitos relacionados con un enfoque intercultural de la enseñanza de la H, G y CCSS. En cuanto a las estrategias de comparación, se busca ayudar a que los estudiantes chilenos y extranjeros reconozcan tanto las características en común, como las especificidades de los procesos históricos de sus respectivos países de origen. En el caso de las analogías, se desea facilitar la comprensión de contenidos que podrían resultar ajenos y poco significativos para los alumnos inmigrantes (Poblete y Galaz, 2016).

\subsection{Las prácticas docentes que se apartan del enfoque intercultural}

Paralelamente a las prácticas docentes descritas, es posible apreciar que los profesores realizan análisis y explicaciones en los que se advierten ideas que se alejan del concepto de "interculturalidad". Algunas de estas ideas se relacionan con conceptos esencialistas de "identidad", narrativas de excepcionalidad nacional y con una visión de superioridad cultural asociada a rasgos tales como la religión. A continuación, presentamos dos ejemplos:

- Identidad esencialista y narrativa de excepcionalidad nacional. El caso de los libertadores

Profesor: como bien señalaba la compañera, esas controversias dependen de quién es el libertador, Bueno perfectamente... nosotros hoy día tendemos a decir que nuestro libertador no sé... Bernardo O'Higgins, los hermanos Carrera.

Alumno: Manuel Rodríguez.

Profesor: Manuel Rodríguez, y nos olvidamos bastante de San Martín que fue clave, eso pasa... eso también pasa con discursos, discursos nacionalistas posteriores a los procesos iya? Finalmente, a mí lo que 
me interesa como chileno es enaltecer la figura de los chilenos y no de los argentinos. (DC, C5)

- Superioridad cultural. Comparación de la cultura musulmana y la cultura cristiana occidental

Estudiante: ¿la religión católica tiene restricciones?

Profesora: sí, pero no tantas como el islam.

Estudiante: y ¿cuáles son una de esas?

Profesora: por ejemplo, una restricción es como lo que te comentaba de la mujer, cómo tiene que vestirse o cómo, a cierta edad, la mujer no puede mostrar su cuerpo, por pudor, se tiene que casar a cierta edad también, tiene muchas limitaciones. En la iglesia católica, tiene hay más bien prejuicios ¿cierto?, relacionados de repente con la homosexualidad o con el género en sí, pero esto es mucho más estricto.

(...)

Profesora: por esa razón el islam es tan restringido Josefina [nombre ficticio], porque, por definición, imagínate, significa sumisión a la voluntad de Alá, ya ni siquiera que tú creas en él, sino que seas una persona sumisa bajo el amparo de Alá.

Estudiante: eso es tóxico profe.

Profesora: exacto, sigamos con la lectura María López [nombre ficticio] siga... ya, en Medina (...). (DA, C3)

En el caso de los libertadores, el profesor plantea una correspondencia entre historia nacional y héroes, y, además de esto, se refiere al interés de enaltecer la figura de los héroes de la propia nacionalidad, en desmedro de los de otras nacionalidades. Ello muestra un concepto de identidad nacionalista y una narrativa de excepcionalidad nacional, por cuanto cada país tiene héroes "exclusivos" a los que buscará darles relevancia, aunque existan héroes como San Martín, que podrían ser referentes identitarios compartidos por más de un país.

En tanto, las características que la profesora resalta de la religión musulmana están marcadas por sesgos negativos, ya que constantemente la compara con el catolicismo, etiquetándola como una religión más restrictiva. Esta idea de "superioridad" resulta particularmente necesaria de cuestionar en la enseñanza de la $\mathrm{H}$, 
G y CCSS, porque está presente en los proyectos interculturales latinoamericanos que, hasta hoy en día, "parten de la diferencia colonial que ha determinado relaciones de subalternidad con distintos pueblos" (Diez, 2004, p. 195).

\section{Consideraciones finales. ¿Desde dónde partir para profundizar el enfoque intercultural de la enseñanza de la H, G y CCSS?}

Sin lugar a duda, los profesores de Historia estudiados muestran en sus prácticas propósitos relacionados con un enfoque intercultural de la enseñanza de la H, G y CCSS. Adicionalmente, estos profesores despliegan una sofisticada capacidad de tomar decisiones pedagógicas, pertinentes al contexto en el que se desempeñan, para propiciar el aprendizaje de todos los estudiantes, lo que corresponde a una de las habilidades más frecuentemente relacionadas con un alto desempeño docente. No obstante, muchas de sus explicaciones están permeadas por ideas que van en sentido opuesto a la interculturalidad. De hecho, basándonos en Nordgren y Johansson (2015), en estos ejemplos se encuentran comprometidas las habilidades de descentrarse e interpretar representaciones de otras culturas, y conectar dichas representaciones con su propia cultura, tomar otras posiciones y mediar entre perspectivas diversas.

A nuestro juicio, esta disonancia podría explicarse por la tensión que se produce entre la necesidad de generar estrategias nuevas para afrontar un contexto escolar inédito y, al mismo tiempo, mantener el rigor conceptual y realizar un análisis de los propios discursos, sin contar con el tiempo ni las ayudas necesarias para ello. Lo anterior se traduce en la falta de instancias en las cuales los docentes puedan cuestionar, en sus prácticas y discursos, lo que la interculturalidad crítica denomina la "matriz de poder, diferenciación y subalternización". Como corolario de ello, no se produce en las clases de H, G y CCSS una reflexión crítica sobre lo que se identifica como "formas culturales propias" (Stefoni et al., 2016) y el enfoque intercultural de la enseñanza en el área se ve limitado. 
En definitiva, una de las necesidades más urgentes, en cuanto a la implementación de una enseñanza de la H, G y CCSS con enfoque intercultural, es la creación de instancias en las cuales los profesores tengan la oportunidad de repensar sus discursos y mejorar sus prácticas, para implementar un concepto más profundo de interculturalidad en los contextos escolares específicos en los que se desempeñan. Siguiendo a Stefoni et al., es fundamental entender que, en muchos casos, este proceso no es espontáneo; por ello, es necesaria una política intercultural que procure "intervenir en el proceso orientándolo hacia espacios de comunicación que no se generarían naturalmente" (Stefoni, et al., 2016, p. 159).

Desde una perspectiva intercultural, la llegada de inmigrantes otorga la oportunidad de revisar y cuestionar la legitimidad de las narrativas nacionales esencialistas y los supuestos de superioridad sobre los cuales se han sustentado las relaciones de desigualdad (Walsh, 2009; Diez, 2004). Este cuestionamiento es pertinente, porque permite cambiar el foco desde un propósito de convivencia armónica entre migrantes y no migrantes, hacia la construcción de un proyecto identitario nacional elaborado en conjunto, asentado en bases más justas y con mayor igualdad, lo que hoy en día es la principal preocupación de la sociedad chilena.

\section{Referencias}

Angvik, M. \& Borries, B. V. (1997). Youth and history. A comparative European survey on historical consciousness and political attitudes among adolescents. Hamburgo: Alemania.

Barton, K. \& Levstik, L. (2004). Teaching history for the common good. New Jersey, London.

Bonilla-García, M. (2016). Ejemplificación del proceso metodológico de la teoría fundamentada. Cinta de Moebio: Revista de Epistemología de Ciencias Sociales, 57, 305-315.

Carretero, M. \& Voss, J. (1994). Cognitive and instructional processes in history and social sciences. New Jersey, United States of America: LEA.

Carretero, M. \& Voss, J. (1998). Learning and Reasoning in History. Vol 2. London: Woburn Press. 
Cid, G. (2012). La nacion bajo examen:la historiografía sobre el nacionalismo y la identidad nacional en el siglo XIX chileno. Polis, 11(32), 329-350.

Departamento de Extranjería y Migración [DEM]. (2017). Circular Nº16. Instruye sobre la visación temporaria para niños, niñas y adolescentes. Santiago, Chile.

Dietz, G. (2012). Multiculturalismo, interculturalidad y diversidad en educación. Una aproximación antropológica. México: FCE.

Diez, M. (2004). Reflexiones en torno a la interculturalidad. Cuadernos de Antropología Social, (19), 1-12.

Fundación Superación de la Pobreza e Instituto de Estudios Municipales (2016). Educación e Interculturalidad en Escuelas Públicas. Orientaciones desde la Práctica. Santiago: FSP. Recuperado de http://www2. superacionpobreza.cl/wp-content/uploads/2019/06/MV_02_EstudioMetropolitana_02_Pra\%CC\%81cticas-de-educacio\%CC\%81nintercultural.pdf

Garcés, A., Riedemann, A., Naudon, P. y Palma, J. (2017). Mesa para una educación intercultural. Hacia una educación intercultural: propuestas para la reflexión-acción en contexto migratorio. Santiago de Chile.

Gregorini, V. (2015). La enseñanza de la historia. Una compleja relación entre norma y práctica. Revista Brasileira de Educación, 61, 457-478.

Hajisoteriou, C., Karousiou, C. \& Angelides, P. (2018). Successful components of school imrpovement in culturally diverse schools. School Effectiveness and School Improvement, 29, 91-112.

Hawley, T., Crowe, A. \& Mooney, E. (2016). Visualizing Social Justice: Using Controversial Images in Social Studies Classrooms. The Clearing House: A Journal of Educational Strategies, Issues and Ideas, 89(3), 85-90.

Ho, L., McAvoy, P., Hess, D. \& Gibbs, B. (2017). Teaching and Learning about Controversial Issues and Topics in the Social Studies. In M. Megan \& M. Cheryl, The Wiley Handbook of Social Studies Research (pp. 321-335). USA: Wiley-Blacjwell.

Hornberg, S. (2002). Human Rights Education as an Integral Part of General Education. International Review of Education, 48(3),187-198.

Hovater, S. (2007). Developing Cultural Awareness: A Grounded Theory Study of Pre-Service Teachers. Field Experiences in Taiwan. Public Access Theses and Dissertations from the Collage of Education and Human Sciences.

Instituto Nacional de Estadística [INE] \& Departamento de Extranjería y Migración [DEM]. (2018). Estimaciones de la población extranjera. Minuta Estimación regional y principales comunas. Santiago, Chile. 
Joiko, S. y Vásquez, A. (2016). Acceso y elección escolar de familias migrantes en Chile: "no tuve problemas porque la escuela es abierta, porque acepta muchas nacionalidades". Calidad en la Educación, (45), 132-173.

Kaplowitz, D., Lee, J. \& Seyka, S. (2018). Looking to near peers to guide student discussions about race. Phi Delta Kappan, 51-55.

Klein, S. (2010). Teaching History in the netherlands: Teaching Experiencies of a Plurality of Perspective. Curriculum Inquiry, 40, 614-624.

Martínez, D. (2018). Estado del arte Migración y Sistema Escolar en Chile, Argentina y España. Santiago de Chile: Centro de Estudios, División de Planificación y Presupuestos, Ministerio de Educación. Recuperado de https://migrantes.mineduc.cl/wp-content/uploads/sites/88/2018/06/ Estado-del-arte-migraci\%C3\%B3n-y-sistemaescolar-en-ChileArgentina-y-Espa\%C3\%Bla.pdf

MINEDUC. (2012). Bases Curriculares para la Educación Básica. Decreto Supremo de Educación No 439/2012. Santiago de Chile: Unidad de Curriculum y Evaluación. Ministerio de Educación, República de Chile.

MINEDUC. (2016). Bases curriculares $7^{\circ}$ básico a $2^{\circ}$ medio. Decreto Supremo de Educación No 614/ 2013. Decreto Supremo de Educación No 369/2015. Santiago de Chile: Unidad de Curriculum y Evaluación. Ministerio de Educación, República de Chile.

MINEDUC. (2017). Orientaciones técnicas para la inclusión educativa de estudiantes extranjeros. Santiago de Chile: División de Educación General. Cordinación Nacional de Inclusión y Diversidad.

MINEDUC. (2018a). Mapa del estudiantado extrajero en el sistema escolar chileno (2015-2017). Santiago de Chile: Documento de Trabajo Nº 12.

MINEDUC. (2018b). Política nacional de estudiantes extranjeros (2018-2022). Santiago de Chile: Mesa Migrante Mineduc. Gabinete Ministerial. División de Educación General.

Mondaca, C., Gajardo, Y. y Sánchez, E. (2014) Historia, memoria y ciudadanía intercultural. El reto del siglo XXI para las aulas de la región de Arica y Parinacota, norte de Chile. Interciencia, 39(7), 524-530.

Nordgren, K. (2017). Poweful knowledge, intercultural learning and history education. Journal of Curriculum Studies, 49(5), 663-682.

Nordgren, K. \& Johansson, M. (2015). Intercultural historical and history education. Journal of Curriculum Studies, 47(1), 2-25.

Poblete, R. (2018). El trabajo con la diversidad desde el currículo en escuelas con presencia de niños y niñas migrantes: estudio de casos en escuelas de Santiago de Chile. Perfiles Educativos, XL(159), 51-65. 
78 INTERCULTURALIDAD Y PRÁCTICAS DOCENTES EN CLASES DE HISTORIA, GEOGRAFÍA Y CIENCIAS SOCIALES, EN ESCUELAS CON ALTO PORCENTAJE DE ALUMNOS MIGRANTES DE LA COMUNA DE SANTIAGO - L. Valledor, L. Garcés, P. Whipple

Poblete, R. y Galaz, C. (2017). Aperturas y cierres para la inclusión educativa de niños/as migrantes en Chile. Estudios Pedagógicos, 239-257

Poblete, R. y Galaz, C. (2016) Informe final de Investigación. Niños y niñas migrantes: trayectorias de inclusión educativa en escuelas de la Región Metropolitana. Santiago de Chile: Superintendencia de Educación de Chile. Recuperado de https://www.supereduc.cl/wp-content/uploads/2017/01/ Informe-Final-de-Investigacio \% CC\% 81 n-Migrantes.pdf

Programa Interdisciplinario de Estudios Migratorios y Fundación Superación de la Pobreza. (2017). Guía Pedagógica para una educación intercultural, anti-racista y con perspectiva de género. Ideas, experiencias y herramientas. Santiago, Chile. Recuperado de https://www.gcedclearinghouse.org/ sites/default/files/resources/170102spa.pdf

República de Chile. (2009). Ley General de Educación. Santiago de Chile: Ley N²9370. Diario Oficial de la República de Chile.

República de Chile. (2015). Ley N²0845. Ley de inclusión escolar que regula la admisión de los y las estudiantes, elimina el financiamiento compartido y prohíbe el lucro en establecimientos educacionales que reciben aportes del Estado. Santiago de Chile: Diario Oficial de la República de Chile.

Riedemann, A. y Stefoni, C. (2015). Sobre el racismo, su negación y las consecuencias para una educación anti-racista en la enseñanza secundaria chilena. Polis, Revista Latinoamericana, 14(2), 191-216.

Salas, N., Castillo, D., SanMartín, C., Kong, F., Thayer, E. y Huepe, D. (2017). Inmigración en la escuela: caracterización del prejuicio hacia escolares migrantes en Chile. Universitas Psychologica, 1-15.

Sandín, P. (2003). Investigación cualitativa en educación. Fundamentos y tradiciones. Madrid: McGraw and Hill Interamericana.

Servicio Jesuita a Migrantes. (2018). Informe de sistematización del Programa Migración y Escuela. Documento de trabajo No1. Santiago, Chile. Recuperado de https://www.academia.edu/37451433/ INFORME_DE_SISTEMATIZACI\%C3\%93N_DEL_PROGRAMA_ MIGRACI\%C3\%93N_Y_ESCUELA_2017

Sheridan, V. \& Storch, K. (2009). Linking the Intercultural and Grounded Theory: Methodological Issues in Migration Research Forum Qualitative Sozialforschung. Quialitative Social Research, 10(1).

Silva, C. y Ballesteros, V. (2017). Reportes Migratorios. Población Migrante en Chile. Santiago de Chile: Ministerio del Interior y Seguridad Pública. Recuperado de https://www.extranjeria.gob.cl/media/2019/04/ReporteMigratorio-Poblaci\%C3\%B3n-Migrante-en-Chile.pdf 
Solhaug, T. \& Osler, A. (2018). Intercultural empathy among Norweigan students: an inclusive citizenship perspective. International Journal of Inclusive Education, 22(1), 89-110.

Stefoni, C., Stang, M. y Riedemann, A. (2016). Educación e interculturalidad en Chile: un marco para el análisis. Estudios Internacionales, 153-182.

Tijoux, M. E. (2013). Las escuelas de la inmigracion en la ciudad de Santiago: Elementos para una educación contra el racismo. Polis, Revista Latinoamericana, 12(35), 287-307.

Walsh, C. (2009). Interculturalidad critica y educacion intercultural. Interculturalidad y educación intercultural, 1-12.

Zembylas, M. \& Kambani, F. (2012). The teaching of controversial issues during elementary -evel history instruction: greek-Cypriot teachers perceptions and emotions. Theory and Research in Social Education, 40(2), 107-133.

Recibido: 16/01/2020

Aceptado: 16/03/2020 


\section{Anexos}

Anexo 1: Pauta de entrevista semiestructurada a docentes

1. ¿Qué te pareció la clase?

2. ¿Por qué elegiste (una guía de aprendizaje, un power point, etc.) como recurso para esta clase?

3. ¿Por qué utilizaste (trabajo en grupos, presentación oral, etc.) como estrategia para esta clase?

4. ¿De qué manera ese recurso y esa estrategia ayuda a que tus estudiantes migrantes y no migrantes aprendan Historia y Ciencias Sociales y se comuniquen entre sí?

5. ¿Por qué implementaste la estrategia (trabajo en grupos, presentación oral, etc.) aplicando el criterio (selección aleatoria de los miembros de los grupos de estudiantes)?

6. ¿Por qué elegiste incluir (las costumbres en bodas, la ropa en fiestas, etc.) de tus estudiantes migrantes como elementos para trabajar el contenido ("la Independencia de Chile", ietc.) en esta clase?

7. ¿Qué te pareció la recepción y motivación que estudiantes chilenos/as y migrantes tuvieron de los recursos didácticos, los contenidos, las estrategias que usaste en la clase?

8. En términos de hacer que tus estudiantes migrantes y no migrantes "se entiendan" y aprendan del otro, ¿qué te gustó de esta clase? ¿Qué mejorarías?

9. ¿Qué te gustaría comentar acerca de cosas que ocurrieron en la clase? 